\title{
Grado de cumplimiento y conocimiento de la resolución 371 de 2009, referente al manejo de medicamentos vencidos en la ciudad de Bogotá, Colombia
}

\author{
Laura Ximena Lobo Berbesí^, Diana Catalina Reina Galindo*^, Luis David \\ Gómez Méndez ${ }^{\star \star}$
}

Universidad Javeriana. Bogotá - Colombia

FeCHa DE ENTREga: 9 DE MAYo DE 2014

FECHA DE EVALUACIÓN: 26 DE MAYO DE 2014

FECHA DE APROBACIÓN: 26 DE MAYO DE 2014

\begin{abstract}
Resumen El presente trabajo tuvo por objeto establecer, mediante observación directa, encuestas (a consumidores, comercializadores y productores de medicamentos) y análisis de datos, el porcentaje de cumplimiento y conocimiento de la Resolución 371 de 2009, por la cual se establece la gestión y disposición final de los medicamentos vencidos, en la ciudad de Bogotá, Colombia.

Dada la naturaleza exploratoria del trabajo, se realizó un sondeo respecto al grado de conocimiento y cumplimiento de dicha resolución, el cual arrojó los siguientes resultados: el $14 \%$ de la población de consumidores conoce y usa los "puntos azules". El $7 \%$ de los comercializadores o distribuidores se preocupa por informar voz a voz el mecanismo para la devolución de medicamentos vencidos. Y el $100 \%$ de los productores o importadores de los medicamentos entrevistados tienen un plan de información a los usuarios sobre la gestión de medicamentos vencidos, solo se entrevistaron a dos empresas productoras o importadoras de medicamentos, del " $n$ muestreal" planteado. Estos resultados muestran un porcentaje inferior al $20 \%$ de cubrimiento de la población, en cuanto a divulgación del plan de gestión de devolución de medicamentos vencidos en la ciudad de Bogotá, como lo exige la norma.
\end{abstract}

\begin{abstract}
This paper aimed to establish - through direct observation, surveys (to consumers, distributors, and producers of medications) and data analysis - the compliance and knowledge percentage of the Resolution 371 of 2009, through which the management and disposal of expired medications in the city of Bogotá, Colombia is established.

Due to the exploratory nature of the paper, we carried out a survey regarding the degree of compliance and knowledge of such resolution. This survey showed the following results: $14 \%$ of consumers knows and
\end{abstract}

\footnotetext{
* Microbióloga Industrial. lauralobob@gmail.com

** Microbióloga Industrial. dcatar90@hotmail.com

$\star \star \star$ Magister en Microbiología. luis.gomez@javeriana.edu.co
} 
uses the "blue points". $7 \%$ of distributors concerns about informing by word of mouth the procedure used to return expired medications. $100 \%$ of producers or importers have a plan to inform users about the handling of expired medications. Only two companies out of the suggested " $n$ sampling" that produce and import medications were interviewed. These results display a percentage below $20 \%$ of population coverage regarding the awareness of the expired medication return management plan in the city of Bogotá, as the regulation establishes.

Palabras Clave: medicamentos vencidos, puntos azules, residuos pos consumo.

Keywords: expired medications, blue points, post-consumption waste.

\section{Introducción}

En el contexto mundial, la industria farmacéutica es considerada como uno de los sectores de mayor relevancia, pues no sólo promueve el desarrollo tecnológico y científico con la permanente implementación de procesos industriales innovadores y la consecuente formulación y producción de medicamentos que responden a gran la diversidad de necesidades de los consumidores; sino que desde el punto de vista económico la participación de 25 sus compañías multinacionales representa el $50 \%$ del mercado mundial, que en 2004 generó ventas de 550.000 millones de dólares [16].

Por otra parte, desde una perspectiva ecológica, la cadena de producción de esta industria, genera diversos tipos de residuos que normalmente reportan una baja Demanda Biológica de Oxígeno (DBO), Demanda Química de Oxígeno (DQO) y un pH cercano a la neutralidad [6]. Cuando estos residuos son sometidos a un proceso de tratamiento previo a su vertimiento a los cuerpos de agua, no representan un riesgo significativo para el medio ambiente ni la salud pública. Sin embargo, el aumento de la producción y consumo de medicamentos es directamente proporcional a su persistencia en los efluentes de las plantas de tratamiento. Estos resultados han despertado el interés por determinar los posibles impactos de dichos residuos en los ecosistemas, especialmente en los peces y mamíferos marinos $[2,3,4,7,11,15,17]$.

En el caso particular de Colombia, la contaminación del medio ambiente por la presencia de medicamentos se conjuga con su inadecuada disposición final en el servicio público domiciliario de aseo que, por ser conglomerados a cielo abierto en un relleno sanitario, con otro tipo de residuos tales como: materia orgánica, plásticos, vidrio, pilas, baterías, entre otros, favorece su transformación en compuestos recalcitrantes, de difícil degradación natural y, por ende, persistentes en la naturaleza. Por esta razón, dichos compuestos son considerados como residuos peligrosos o RESPEL y deben ser incluidos en un sistema de disposición final, como lo sugiere la Política Ambiental Nacional [13].

Dicha Política Ambiental Nacional surge como herramienta ante la necesidad de sistematizar la información relacionada con el origen, cantidad, calidad, manejo y gestión de los residuos peligrosos, pues, hasta el momento sólo existían estudios 
aislados y sectorizados que no reflejaban la situación actual de dichos residuos $[5,11]$.

Lo anterior, sumado a la falta de planeación de su disposición final, normatividad dispersa en la que se plantea un manejo orientado a las acciones correctivas y no a la implementación de procesos industriales más limpios, desconocimiento de los impactos negativos por parte de los generadores y receptores, ausencia de reglamentos técnicos que faciliten su clasificación, identificación y caracterización, así como una oferta limitada de laboratorios especializados en el tratamiento de dichos residuos, estimulan la creación de dicha Política Ambiental Nacional, la cual tiene por alcance la gestión de residuos sólidos, semisólidos, líquidos y gases contenidos para su manejo y gestión en las diferentes etapas de manejo como: generación, transporte, comercialización y distribución, consumo, almacenamiento, aprovechamiento, tratamiento, disposición final, importación y exportación [13].

En respuesta al cumplimiento de dicha Política, en el 2009 se promulgó la Resolución 371, por medio de la cual se "establecen los elementos que deben ser considerados en los Planes de Gestión de Devolución de Productos Posconsumo de Fármacos o Medicamentos Vencidos" [11].

Con el fin de asegurar la recolección de los medicamentos vencidos, dicha Resolución plantea que en el primer año a partir de su publicación, se implemente un plan piloto de recolección en el cual se incluyan actividades de sensibilización al consumidor y a los actores de la cadena. A partir del tercer año debe presentar anualmente aumentos del $10 \%$ de cubrimiento de la población, hasta lograr un $70 \%$ de cubrimiento en el país.

El objetivo del presente trabajo fue establecer mediante observación directa, encuestas y análisis de datos, el porcentaje de cumplimiento de la Resolución 371 de 2009, por la cual se establece la gestión y disposición final de los medicamentos vencidos, en la ciudad de Bogotá.

\section{Materiales y métodos}

\subsection{Determinación del tamaño de la muestra poblacional}

En un primer momento se determinó el tamaño de las muestras de los tres tipos de poblaciones a encuestar: personas del común o consumidores, comercializadores o distribuidores y productores o importadores de los medicamentos, aplicando la siguiente fórmula para cada caso:

$$
n=\frac{Z^{2}(p)(q)}{e p^{2}+Z^{2} \frac{(p)(q)}{N}}
$$

En donde:

- $Z$ = Valor de $z$, valor de confianza dado por la tabla de distribución normal.

- $e p=$ Error proporcional, describe qué tan reproducibles son las mediciones.

- $p=$ proporción, de cumplimiento de dicha medición.

- $q=$ proporción, de que no se cumpla dicha proporción. 
- $N=$ Tamaño de la población

En cada población muestreal, se trabajó con un valor de confianza de un $95 \%$ y un error proporcional del $5 \%$. Para la realización de las encuestas se aplicó un muestreo aleatorio estratificado, debido a que el tamaño de cada muestra era variable. En el caso de los consumidores finales, se seleccionaron personas mayores de edad o jefes de hogar, que se encontraran en el perímetro de los "puntos azules", dispositivos usados para la disposición temporal de los medicamentos vencidos, ubicados en las farmacias de supermercados de grandes superficies o de barrios.

Con el fin de determinar la población a encuestar, se utilizó la base de datos del Departamento Nacional de Estadística (DANE), en donde se obtuvo que para el 15 de julio de 2010, la población de Bogotá que cumplía con las características mencionadas era de 1'700.000 habitantes. Al aplicar la fórmula se obtuvo un "n-muestreal" de 283 personas.

Para el caso de los fabricantes de medicamentos, se seleccionaron de manera aleatoria aquellas empresas que se autorizados por el Instituto Nacional de Vigilancia de Medicamentos y Alimentos -INVIMA- al 15 de Julio de 2010. Se escogieron aquellos fabricantes ubicados en la ciudad de Bogotá. Se obtuvo un " $n$-muestreal" de 20 fabricantes y/o importadores.

En el caso de los distribuidores y /o comercializadores se seleccionaron de manera aleatoria aquellas droguerías que tuvieran un "punto azul" registrado por la Asociación Nacional de Industrias -ANDI- al 15 de julio de 2010. El " $N$-poblacional" era de 155 , después de realizada la formula se obtuvo un " $n$ muestreal" equivalente a 40.

\subsection{Planteamiento de las encuestas para cada población}

En el caso de los tres segmentos de las poblaciones encuestadas, se tomaron como punto de referencia las directrices estipuladas por la Resolución 371 de 2009. Para las encuestas dirigidas a los fabricantes y/o generadores de medicamentos, se tuvieron en cuenta las disposiciones establecidas por los artículos 10 y 21 del Decreto 4741 de 2005, relacionadas con su obligación de garantizar la gestión y manejo integral de dichos residuos mediante un plan documentado que prevenga la generación y reducción en la fuente, además de minimizar la cantidad y peligrosidad de los mismos. (Decreto 4741 de 2005)

Así mismo, se formularon las preguntas en función del artículo 8 del Decreto 2676 de 2000, y de la Resolución 1164 de 2002, referentes a las obligaciones del generador de residuos hospitalarios y similares, que incluyen los medicamentos y/o fármacos vencidos, parcialmente consumidos, deteriorados, alterados y/o excedentes (Decreto 2676 de 2000 Resolución 1164 de 2002).

\section{Resultados y discusión}

Con el fin de llevar a cabo las encuestas a la población de consumidores y distribuidores y/o comercializadores de medicamentos de la ciudad de Bogotá, 
se seleccionaron las localidades de Usaquén, Chapinero, Kennedy y Usme, que fueron las cuatro zonas en las cuales se implementaron y desarrollaron los planes piloto en 2009.

\subsection{Datos obtenidos de la encuesta realizada a consumidores}

La encuesta realizada para los consumidores tenía como objetivo evidenciar el grado de conocimiento y uso de los "puntos azules", información que se convierte en un indicativo directo de la efectividad de la implementación de los mecanismos de comunicación a los consumidores acerca de los planes piloto de la gestión de devolución de fármacos y/o medicamentos vencidos por parte de los fabricantes y/o productores y los comercializadores y/o distribuidores, con el objetivo de lograr la cobertura anual porcentual, incluyendo medidas preventivas de riesgos en la salud y el medio ambiente como lo establece la Resolución 371 de 2009 [11].

Dentro de los resultados obtenidos, se encontró que un $60 \%$ de las personas del común preferían desechar los medicamentos vencidos o sus residuos en la basura; un $26 \%$ en el drenaje, y sólo un $14 \%$ de esta población utilizaba los puntos azules (Figura 1). Lo anterior, permite inferir que posiblemente los mecanismos adoptados para la divulgación al consumidor, acerca del los "puntos azules", no son adecuados para alcanzar la cobertura de información a la población del $20 \%$ en la ciudad de Bogotá, como lo sugiere dicha norma al tercer año de su publicación, ya que la población continúa descartando de manera inadecuada los medicamentos.

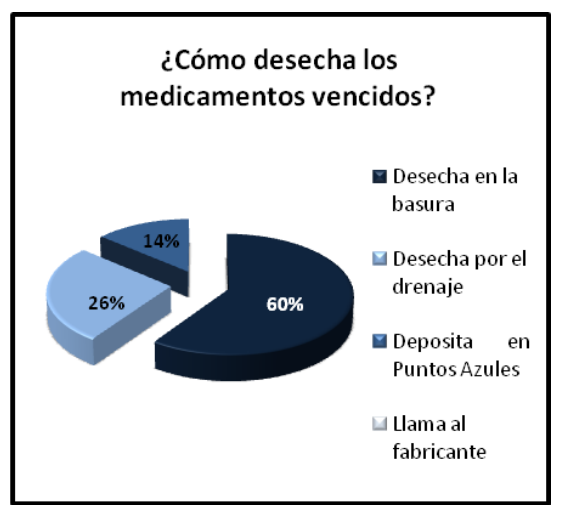

Figura 1. Forma más común adoptada por la población para desechar los medicamentos vencidos.

Sin embargo, los datos obtenidos contrastan con el reporte realizado por la Asociación Nacional de Empresarios de Colombia -ANDI-, el cual indica que en los años 2010 y 2011 se recolectaron 21.8 toneladas de medicamentos vencidos a través de la instalación de 311 contenedores, logrando una cobertura del $10.22 \%$ 
en la población, superando la meta planteada en la Resolución 371 de 2009, equivalente al $10 \%$ para el primer año de implementación y un $20.5 \%$ para el segundo año [8].

Cabe resaltar que la presente encuesta se realizó en el año 2011, por lo que probablemente los datos obtenidos reflejan la incipiente campaña de sensibilización a la población. La anterior inferencia se confirma con la respuesta equivalente al $15 \%$ de la población que afirmó conocer los "puntos azules" (Figura 2) y su ubicación en farmacias y supermercados de grandes superficies. En contraste un $85 \%$ manifestó no poseer información al respecto, lo que refleja desinformación de gran parte de la población acerca de la importancia de recolectar este tipo de medicamentos en los lugares establecidos por las entidades responsables, Igualmente se encontró que del $15 \%$ de la población que afirmaba conocer los puntos azules, por lo menos el $7 \%$ nunca los utilizaba argumentando que no estaban ubicados cerca a su domicilio.

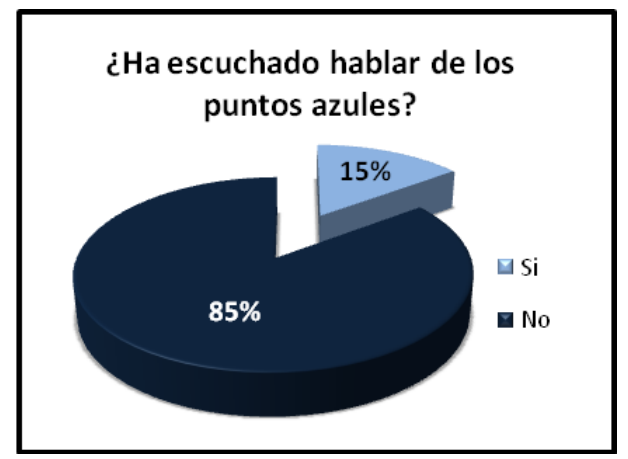

Figura 2. Porcentaje de conocimiento respecto a su uso por parte de los consumidores.

Igualmente, estos resultados demuestran la falta de compromiso que cada individuo tiene con el medio ambiente y la salud de la población, así como las posibles fallas en la divulgación del tema, ya que la mayoría de los encuestados consideraban que los medicamentos vencidos no generan un impacto negativo sobre el ambiente, pues asumen que éstos sufren un proceso de dilución y autodepuración natural. Se sabe, sin embargo, que a bajas concentraciones, los remanentes de dichos productos pueden generar impactos de fertilidad, mortandad y mutaciones en los organismos que conforman los ecosistemas acuáticos y por ende en la cadena trófica, por bioacumulación [14].

Las preguntas 3 y 4 referentes a la información que posee la población sobre la función de los centros de acopio de medicamentos vencidos y su ubicación, son de carácter complementario pues permiten determinar la veracidad de las respuestas anteriores y establecer que sólo el $19 \%$ de la población encuestada sabe qué es y para qué sirve un centro de acopio temporal de medicamentos y/o fármacos vencidos (Figura 3), lo cual concuerda con los resultados obtenidos en 
cuanto al conocimiento de la ubicación de los "puntos azules". Esto indica que sólo el $35 \%$ de la población encuestada, sabe que estos centros se encuentran principalmente en supermercados de grandes superficies y en algunas cadenas de farmacia; sin embargo esto no significa que la población muestreada use los "puntos azules" (Figura 4).

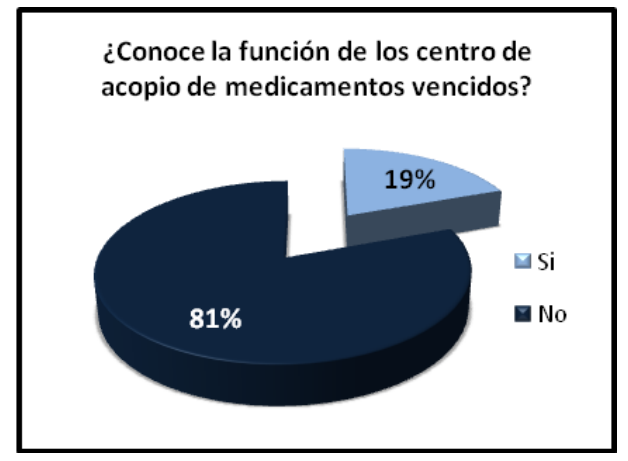

Figura 3. Grado de conocimiento de la función de los centros de acopio de medicamentos vencidos.



Figura 4. Conocimiento que tienen los consumidores sobre la ubicación de los "puntos azules".

Con respecto a las preguntas 5 y 6 referentes a los marcos legales vigentes que rigen la gestión de los medicamentos y/o fármacos vencidos, así como la información sobre la existencia del ente regulador de la gestión de dichos residuos peligrosos, se observa un desconocimiento del $89 \%$ y el $72 \%$ respectivamente, lo que indica que los esfuerzos realizados por el Ministerio del Medio Ambiente para 
dar a conocer la Resolución 371 de 2009, como un marco legal que involucra de manera conjunta a los actores implicados en el ciclo de producción, distribución y consumo de los medicamentos, no han sido lo suficientemente eficientes para lograr el funcionamiento integral de los planes de gestión para la devolución de dichos residuos peligrosos (Figuras 5 y 6 ).



Figura 5. Conocimiento porcentual que tienen los consumidores sobre alguna normatividad referente a la gestión de medicamentos vencidos.

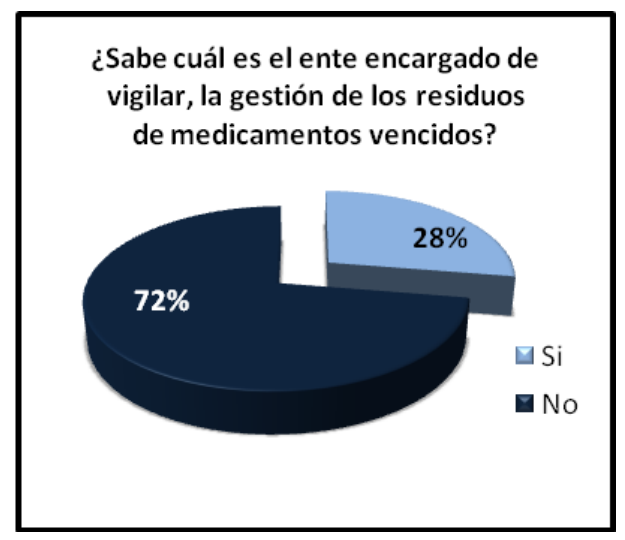

Figura 6. Conocimiento acerca del ente vigilante de la gestión de medicamentos vencidos. 


\subsection{Datos obtenidos a partir de la encuesta realizada a los distribuidores y/o comercializadores que contaban con un "punto azul" en su farmacia}

De acuerdo con los resultados obtenidos en la encuesta realizada a los distribuidores y/o comercializadores de medicamentos, se evidencia un desconocimiento generalizado $(63 \%)$ de la normatividad concerniente a la gestión de medicamentos vencidos (Figura 7), en donde el personal de la farmacia a pesar de tener conocimientos técnicos en auxiliar farmacéutico, tal como lo determina el artículo 18 del Decreto 1950 de 1964, no conoce la Resolución 371 de 2009 ni la importancia de su riguroso cumplimiento.

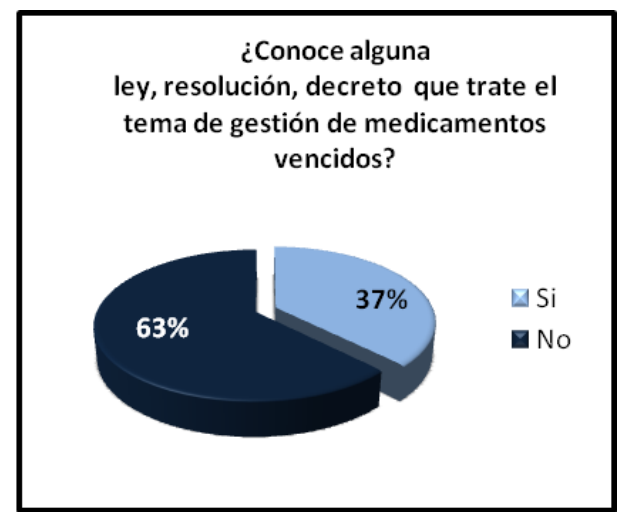

Figura 7. Porcentaje de conocimiento y desconocimiento por parte de los distribuidores y/o comercializadores respecto al marco legal concerniente al tema de gestión de medicamentos vencidos.

Lo anterior se presentó en conjunto con la percepción acerca de la no peligrosidad de los medicamentos vencidos para el medio ambiente y la salud pública, con el argumento de que dichos productos no se clasifican bajo la denominación de "RESPEL" dado que no son reactivos, explosivos o infecciosos (Figura 8). Esto significa que se desconocen no sólo la resolución evaluada en el presente trabajo, sino el Decreto 4741 del 30 de diciembre de 2005 que reglamenta (...) "parcialmente la prevención y el manejo de los residuos o desechos peligrosos generados en el marco de la gestión integral (...)". (Colombia, Decreto 4741 de 2005)

A pesar de que el $60 \%$ del personal a cargo de las farmacias encuestadas recibe cursos periódicos de capacitación, tal como lo contempla el Decreto 4741 de 2005 (Figura 10), se encuentra muy poco interés por establecer sistemas de comunicación y divulgación a los consumidores, acerca de los riesgos y consecuencias que tienen los medicamentos vencidos sobre el medio ambiente y la salud pública, pues únicamente el $7 \%$ de las farmacias encuestadas se preocupan por 


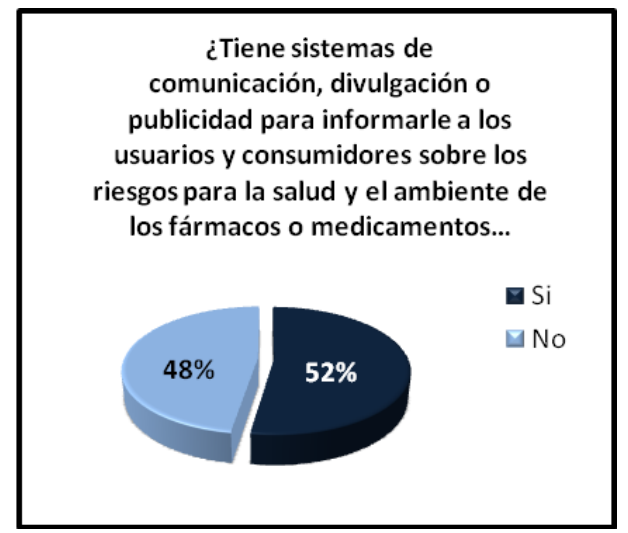

Figura 8. Porcentaje de distribuidores y/o comercializadores encuestados que cuentan con sistemas de comunicación al usuario y/o consumidor, respecto al impacto que tienen sobre el medio ambiente los fármacos y medicamentos vencidos y los riesgos que éstos representan para la salud de la población.

dar información voz a voz a sus clientes, sobre el impacto ambiental generado al desechar en la basura o en el alcantarillado, los medicamentos que han dejado de usar, indicándoles que la correcta disposición de los medicamentos vencidos se realiza en los "puntos azules" ubicados en las farmacias (Figura 9).

Se evidencia así, el incumplimiento de las obligaciones establecidas en la Resolución 371 de 2009, por parte de los distribuidores y/o comercializadores en relación con la adopción de sistemas de divulgación o información a los consumidores para promover el uso de los puntos azules. Esto también se relaciona con la falta de conocimiento por parte de los consumidores acerca de la existencia de estos centros de acopio temporales de los medicamentos vencidos. Es significativo además, que para la mayoría de las farmacias encuestadas la existencia de los "puntos azules" se considera como un requisito legal más, que deben cumplir.

\subsection{Datos obtenidos a partir de la encuesta realizada a los fabricantes y/o importadores de medicamentos de uso humano de la ciudad de Bogotá}

La encuesta estuvo enfocada a la indagación de la presencia e implementación de planes de gestión de disposición final de los medicamentos vencidos, el cual incluía sistemas de monitoreo periódico (mensual) al ambiente, respecto a los impactos generados por los residuos obtenidos del proceso de producción; información al consumidor respecto a la importancia y los riesgos a la salud y al ambiente que puede generar la inadecuada disposición final de los medicamentos vencidos. (Resolución 1164 de 2002)

Sin embargo, los datos obtenidos en las encuestas realizadas a los productores y/o fabricantes de medicamentos de la ciudad de Bogotá, no representan el 




Figura 9. Porcentaje de distribuidores y/o comercializadores encuestados que cuentan con sistemas de comunicación al usuario y/o consumidor, diferentes al volante proporcionado por el Ministerio de Medio Ambiente, respecto a la importancia de la devolución de los medicamentos vencidos en los "puntos azules".

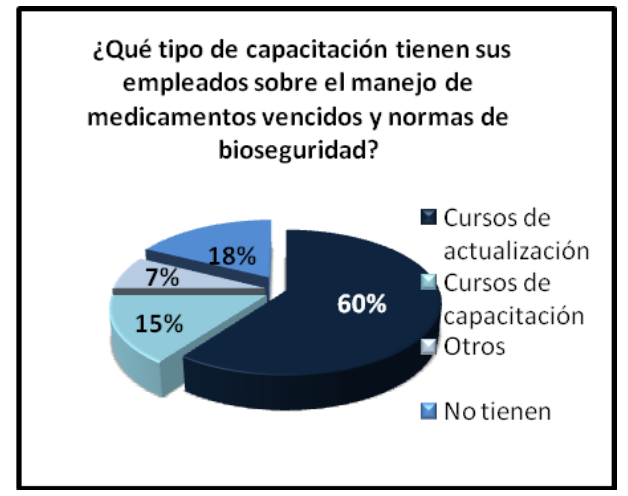

Figura 10. Porcentaje del tipo de capacitación y/o conocimiento del personal de los puntos de distribución y/o comercialización de medicamentos, con respecto al manejo de medicamentos y/o fármacos y normas de bioseguridad.

comportamiento generalizado de la población mencionada, dado que la mayoría de industrias farmacéuticas se mostraron escépticas ante la aplicación de las encuestas, por lo que sólo se entrevistaron a dos productores de medicamentos.

A pesar que los productores o fabricantes de medicamentos encuestados, afirmaron tener posesión e implementación de un plan de información a los usuarios y/o consumidores referente a la devolución de los medicamentos vencidos o remanentes que no sean utilizados en su totalidad, la encuesta realizada a los consumidores, demuestra el desconocimiento que tienen respecto a esta 
posibilidad, ya que el $100 \%$ de las respuestas obtenidas evidenciaron la falta de contundencia de las campañas de información, divulgación y/o comunicación hacia los consumidores, lo cual refleja nuevamente una desarticulación entre la Resolución 371 de 2009 y la situación real en la que se desempeña cada uno de los actores encuestados.

Las estadísticas oficiales indican que para el 2011 la generación de residuos peligrosos fue de 174.418,7 toneladas, cifra superior a las $141.735,0$ toneladas generadas en 2010, e inferior al año 2009, lo cual indica que el amplio marco normativo no ha logrado disminuir significativamente la producción de RESPEL a nivel nacional [8].

Lo anterior, guarda una proporción con el crecimiento económico de un 5,9\% para el 2011 y con la distribución de las categorías de generadores de RESPEL determinado por el Decreto 4741 de 2005, en donde los grandes generadores han aportado un promedio del 92,5\% de la generación total anual, los medianos el $6,3 \%$, los pequeños el 1,1\% y los no obligados el $0,1 \%$ [8].

Según las cifras oficiales, las industrias farmacéuticas presentan la menor participación en la producción de residuos peligrosos, sin embargo, no dejan de ser de interés dado los impactos que generan en la salud humana y su persistencia en el medio ambiente, como es el caso de las 150,3 toneladas anuales de mercurio y sus compuestos producidas, sólo 11,5 son aprovechadas, 38.6 son tratadas y las 81,9 Toneladas restantes sufren el proceso de incineración (disposición final). De acuerdo a las cifras del Perfil Nacional de Sustancias Químicas en Colombia, para el año 2010 se produjeron 1.541,4 Toneladas de desechos y productos farmacéuticos, de los cuales sólo 389,1 Toneladas fueron aprovechadas, 1.294,5 Toneladas fueron tratadas y 121,6 tuvieron una disposición final [14].

\section{Conclusiones}

Se estableció que sólo el $14 \%$ de la población encuestada de las localidades de Chapinero, Kennedy, Usaquén y Usme conocen y usan los "puntos azules", lo cual no cumple con el porcentaje gradual establecido por la Resolución 371 de 2009, equivalente al $20 \%$ para el tercer año, después de la publicación de dicha normatividad. Es importante resaltar que en el presente estudio no fue posible determinar la localidad que poseía el mayor número de "puntos azules", debido a que se utilizó un muestreo aleatorio estratificado, en donde la selección del tamaño de la muestra se estipuló en función del carácter finito, contable y la heterogeneidad de la población de farmacias con "puntos azules" en la ciudad de Bogotá.

Así mismo, no se determinó una reciprocidad entre la cantidad de "puntos azules" por localidad y el mayor o menor grado de conocimiento de la Resolución 371 del 2009, por parte de los consumidores, ya que el diseño de la encuesta no estaba enfocado a determinar que la población encuestada viviera en la misma localidad del "punto azul" encuestado, pues de acuerdo con dicha normatividad, independientemente de la localidad en la que habiten los consumidores finales, se 
debe realizar un cubrimiento de información a la población de Bogotá equivalente al $20 \%$ para el presente año (2012).

Se logró evidenciar que la muestra poblacional de los fabricantes no cumple con la gestión de medicamentos vencidos y planes de divulgación hacia los consumidores como lo establece la Resolución 371 de 2009. Sin embargo, cabe resaltar que los datos obtenidos de los fabricantes y/o productores de medicamentos de la ciudad de Bogotá no son representativos respecto al comportamiento generalizado de los fabricantes.

Se determinó a través de la revisión de la normatividad legal vigente y las estadísticas oficiales que si bien los medicamentos vencidos y demás productos farmacéuticos no son significativos en cuanto a su cantidad, como otros residuos peligrosos, sí representan un peligro para la salud humana y el medio ambiente, dado que su generación ha aumentado a través del tiempo.

Se identificó que la incineración es el tratamiento más utilizado y estipulado por la normatividad legal vigente para la disposición final de los residuos peligrosos, particularmente para los medicamentos y productos farmacéuticos. Sin embargo, aún sigue siendo insuficiente el número de instalaciones autorizadas y la tecnología necesaria para el manejo de los residuos peligrosos a nivel nacional.

\section{Referencias}

1. Asociación Nacional de Empresarios de Colombia -ANDI-. Plan de devolución de medicamentos o fármacos vencidos. 2012. [en línea]. Disponible en: http://www .andi.com.co/archivos/file/Vicepresidencia\%20Desarrollo\% 20Sostenible/ECOPUNTO/PUNTO $\% 20$ AZUL $\% 20-\% 20$ Comunicado $\% 20$ Institucional $\%$ 20V2.pdf. Consultado el 01 de Julio de 2014.

2. Bila M. D., Dezotti M.: Fármacos no medio ambiente. Quím.Nova. 26, 523-530. (2003).

3. Bolong N., Ismail F. A., Salim M., Matsuura, T.: A review of the effects of emerging contaminants in wastewater and options for their removal. Desalination. 239, 229246. (2009).

4. Brain R. A., Hanson M. L., Solomon K. R., Brooks, B. W.: Aquatic plants exposed to pharmaceuticals: effects and risks. Rev Enviro Contam Toxicol . 192,67-115. (2008).

5. DNP-UPA, FIUN-PIRS.: Estudio sobre los Residuos Peligrosos en Colombia: Un Primer Paso Para la Acción. (1998).

6. Environmental Protection Agency -EPA-: Guides to Pollution Prevention the Pharmaceutical industry. (1991).

7. Filho W.R., Barreiro J.C., Vieira E.M. \& Cass Q.B.: Fármacos, ETEs e corpos hídricos. Revista Ambiente \& Agua. 2, 54-61. (2007).

8. IDEAM.: Informe Nacional sobre Generación y Manejo de Residuos o Desechos Peligrosos en Colombia, año 2011. Bogotá, D. C., 62 p. (2012).

9. Jelic, A., Gros, M., Ginebreda, A., Cespedes, R., Ventura, F., Petrovic, M., Barcelo, D.: Ocurrence, partition and removal of pharmaceuticals in sewage water and sludge during wastewater treatment. Water Rest 45, 1165-1176. (2011).

10. Kim Y., Choi K., Jung J., Park S., Kim P.G. \& Park J.: Aquatic toxicity of acetaminophen, carbamazepine, cinetidine, ditiazem and six major sulfonamides, and their potential ecological risks in Korea. Environ Int. 33, 370-375. (2007). 
11. MADS.: Resolución 371. Por medio de la cual se establecen los elementos que deben ser considerados en los planes de gestión de devolución de productos posconsumo de fármacos o medicamentos vencidos. Ministerio del Medio Ambiente y Desarrollo Sostenible. Diario oficial. 6 de febrero de 2009. (2009).

12. MADS.: Resolución 1164. Por la cual se adopta el manual de procedimientos para la gestión integral de los residuos hospitalarios y similares. Ministerio de Medio Ambiente y Desarrollo Sostenible. Diario oficial. 6 de septiembre de 2002. (2002).

13. MADS.: Decreto 4741. Por el cual se reglamenta parcialmente la prevención y el manejo de los residuos o desechos peligrosos generados en el marco de la gestión integral. Ministerio de Medio Ambiente y Desarrollo Sostenible. Diario oficial. 30 de diciembre de 2005. (2005).

14. López Arias, Andrea; Suárez Medina, Oscar J.; Hoyos Martha C.: Perfil Nacional de Sustancias Químicas en Colombia. 2da. Ed. - Bogotá, D.C.: Colombia. Ministerio de Ambiente y Desarrollo Sostenible; Naciones Unidas para el Desarrollo Industrial - UNIDO, (2012).

15. Sarmah A. K., Meyer M.T., Boxall A.B.: A global perspective on the use, sales, exposure pathways, occurrence, fate and effects of veterinary antibiotics (VAs) in the environment. Chemosphere. 65, 725-759. (2006)

16. Vásquez V.J.: El sector farmacéutico en el marco de las negociaciones del Tratado de Libre Comercio con los Estados Unidos. Perfil de Coyuntura Económica. 5, 33-43. (2005).

17. Yu T., Yu-Chen A., Panchangam S., Andy P., Yang P., Lin, C.: Biodegradation and bio-sorption of antibiotics and non-steroidal anti-inflammatory drugs using immobilized cell process. Chemosphere. 84, 1216-1222. (2011). 